\title{
Inclusive and Exclusive Spin Structure Measurements in the Resonance Region
}

\author{
G.E. Dodge for the CLAS Collaboration \\ Department of Physics, Old Dominion University, Norfolk, VA 23529
}

\begin{abstract}
A program of spin structure function measurements is underway in Jefferson Lab's Hall B. We use polarized electrons incident on polarized $\mathrm{NH}_{3}$ and $\mathrm{ND}_{3}$ targets to study proton and deuteron spin observables in and above the resonance region. Results from the first set of data taken in 1998 will be presented for the first moment of $g_{1}$ and for the double polarization asymmetry for exclusive pion production, which is sensitive to the spin structure of the nucleon resonances.
\end{abstract}

\section{MOTIVATION}

The spin structure functions $g_{1}$ and $g_{2}$ of the nucleon have been a topic of great interest for more than two decades. These structure functions have been extensively measured in deep inelastic scattering (DIS) experiments over a wide kinematic range in Bjorken $x$ and momentum transfer $Q^{2}$. Particularly interesting is the first moment of $g_{1}, \Gamma_{1}=\int_{0}^{1} g_{1}\left(x, Q^{2}\right) d x$, which is related to the spin carried by the quarks at large $Q^{2}$. Comparisons between these data and theoretical predictions, especially the fundamental Bjorken Sum Rule [1], have yielded reasonable agreement.

At the real photon point, $Q^{2}=0$, the Gerasimov Drell Hearn (GDH) Sum Rule [2] relates the difference of the cross section for photo absorption of a linearly polarized photon antiparallel and parallel to the spin of a nucleon to the anomalous magnetic moment of the nucleon, $\kappa_{N}$. The slope of $\Gamma_{1}$ at low $Q^{2}$ is constrained by the value of the GDH Sum Rule, $\Gamma_{1}\left(Q^{2}\right)=-\frac{Q^{2}}{8 M^{2}} \kappa_{N}^{2}$. Since $\Gamma_{1}$ is negative at low $Q^{2}$ and positive in the DIS regime, it must have an interesting behavior as a function of $Q^{2}$, including a sign change. An intense experimental effort is now underway at DESY and Jefferson Lab to investigate the low to moderate $Q^{2}$ regime, where resonances are expected to play an important role.

\section{EXPERIMENT}

At the Thomas Jefferson Laboratory in Newport News, Virginia, an extensive investigation of spin structure functions at low to moderate $Q^{2}$ is underway in all three experimental halls. In Hall B, our experimental program encompasses measurements of the double polarization asymmetry for both inclusive and exclusive processes on the proton and the deuteron. We scatter longitudinally polarized electrons from longitudinally polarized $\mathrm{NH}_{3}$ and $\mathrm{ND}_{3}$ targets and detect the reaction products in the CEBAF Large 


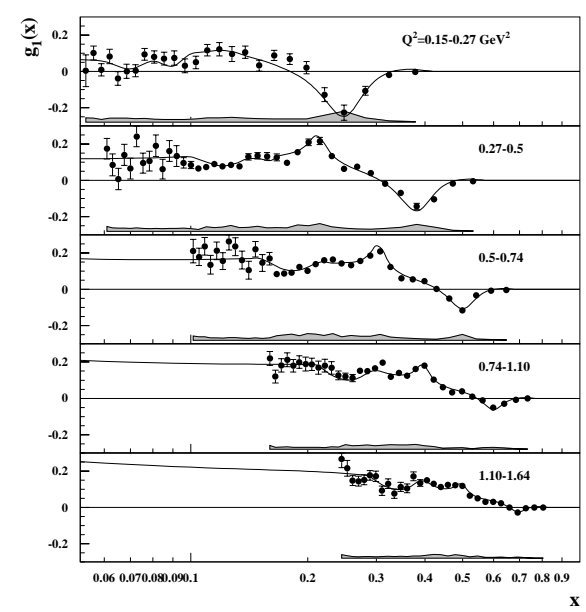

FIGURE 1. $g_{1}$ for the proton in five $Q^{2}$ bins. The solid line is our parameterization of world data and the shaded band indicates the systematic error.

Acceptance Spectrometer (CLAS). The electron polarization is measured with a Moller polarimeter and the target polarization is monitored with an NMR system. In the fall of 1998 we recorded three billion triggers with beam energies of 2.5 and $4.2 \mathrm{GeV}$. For seven months in 2000 and 2001 we took an additional 23 billion triggers at beam energies of 1.6, 2.5, 4.2 and 5.7 GeV. Results from the 1998 data are presented here.

\section{INCLUSIVE ANALYSIS AND RESULTS}

The raw asymmetry for longitudinally polarized beam and target is given by $A_{\text {raw }}=$ $\left(N^{\uparrow \downarrow}-N^{\uparrow \uparrow}\right) /\left(N^{\uparrow \downarrow}+N^{\uparrow \uparrow}\right)$ where $N^{\uparrow \downarrow}\left(N^{\uparrow \uparrow}\right)$ is the yield normalized to the accumulated electron charge for beam and target antiparallel (parallel). The raw asymmetry must be divided by the dilution factor $f$, which corrects the denominator for target nuclei which are not polarized. After applying radiative corrections, the asymmetry

$$
A_{1}+\eta A_{2}=\frac{1}{D P_{e} P_{t} f} A_{\text {raw }}, \text { where } D=\frac{1-\varepsilon E^{\prime} / E}{1+\varepsilon R} \text { and } \eta=\frac{\varepsilon \sqrt{Q^{2}} / E}{1-\varepsilon E^{\prime} / E},
$$

can be extracted. $A_{1}$ is the transverse photo absorption asymmetry and $A_{2}$ is the ratio of the longitudinal/transverse interference cross section to the total transverse cross section. The product of the electron beam $P_{e}$ and target $P_{t}$ polarization is determined by comparing our measured asymmetry in the elastic peak to known values. Finally, we extract $g_{1}$

$$
g_{1}\left(x, Q^{2}\right)=\frac{\tau}{1+\tau}\left[A_{1}\left(x, Q^{2}\right)+\frac{1}{\sqrt{\tau}} A_{2}\left(x, Q^{2}\right)\right] F_{1}\left(x, Q^{2}\right),
$$




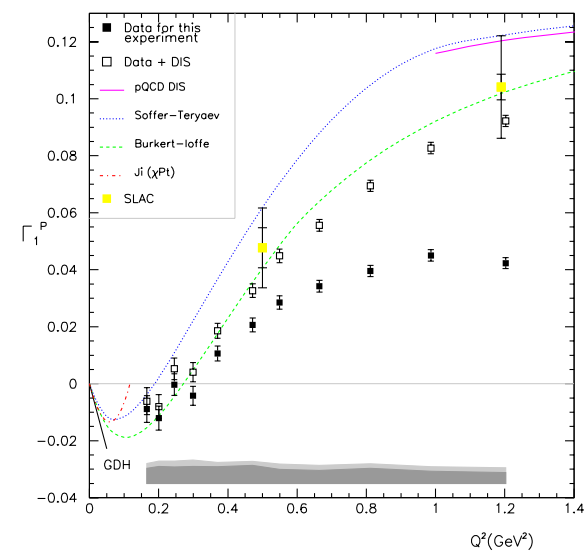

FIGURE 2. $\Gamma_{1}^{p}$ as a function of $Q^{2}$ integrated over our measured range in $x$ (solid squares) and including an extrapolation to $x=0$ (open squares). The systematic error on our measured integral is shown by the dark grey band and the additional contribution from the extrapolation is indicated by the light grey band. Models by Burkert and Ioffe [3] and Soffer and Teryaev [4] are shown as dashed and dotted lines. SLAC data [5] are plotted as the shaded squares. The Chiral Perturbation calculation of Ji [6] is indicated by the dot dashed line.

where $\tau=v^{2} / Q^{2}$, using a model which includes a parameterization of world data for $A_{2}$ and the unpolarized structure function $F_{1}$.

Figure 1 shows $g_{1}\left(x, Q^{2}\right)$ for the proton for several bins in $Q^{2}$. The resonant structure is clearly visible, including the negative delta resonance at large $x$, which decreases as $Q^{2}$ increases. The solid line indicates our model, which has been tweaked slightly to accomodate our data. This same model was used to extrapolate to the unmeasured DIS region at low $x$ in order to calculate the integral $\Gamma_{1}^{p}$ shown as the open squares in Figure 2. The solid squares indicate the contribution to the integral from the range in $x$ that we measure, which includes the resonance region up to at least $W=2 \mathrm{GeV}$ for all $Q^{2}$ bins. The data change sign at $Q^{2} \sim 0.25 \mathrm{GeV}^{2}$ and seem to favor the phenomenological model of Burkert and Ioffe [3] which explicitly includes resonance contributions on top of a smooth curve connecting the real photon point to the high $Q^{2}$ regime.

From the $\mathrm{ND}_{3}$ data we have extracted $\Gamma_{1}^{d}$, shown in Figure 3. The solid triangles indicate the contribution to the integral that we measure and the open triangles include the extrapolation to the DIS regime. In addition to the predictions by Burkert [3] and Soffer [4], the contribution to the integral from resonances alone, as calculated by the AO model [7], is shown as the short dashed line. The sign of $\Gamma_{1}^{d}$ changes at roughly $0.6 \mathrm{GeV}^{2}$ and follows the trend of the various models. The statistical precision of the 1998 deuteron data is not as good as for the proton because the target polarization was poor and consequently less data were taken on the deuteron target. Analysis of the much larger 2000-2001 EG1 data set is underway; preliminary results are presented elsewhere in these proceedings [8]. 


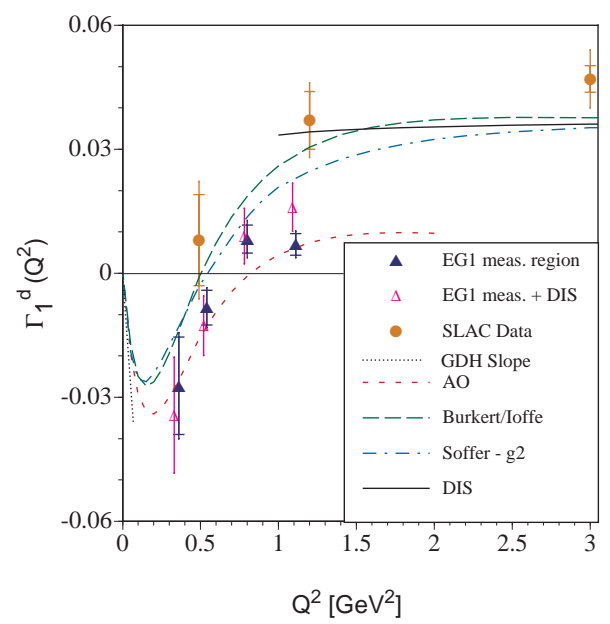

FIGURE 3. $\Gamma_{1}^{d}$ as a function of $Q^{2}$ integrated over our measured range in $x$ (solid triangles) and including an extrapolation to $x=0$ (open triangles). Models by Burkert and Ioffe [3] and Soffer and Teryaev [4] are shown as long-dashed and dash-dotted lines. SLAC data [5] are shown as the filled circles. The resonance contribution shown by the short dashed line is from the AO code [7].

\section{EXCLUSIVE ANALYSIS AND RESULTS}

There is also a great deal of interest in the study of polarization observables in exclusive reactions. The cross section $\sigma$ for an exclusive reaction can be written as

$$
\sigma=\sigma_{0}+P_{e} \sigma_{e}+P_{t} \sigma_{t}+P_{e} P_{t} \sigma_{e t}
$$

where $\sigma_{0}$ is the unpolarized cross section and $\sigma_{e}, \sigma_{t}$ and $\sigma_{e t}$ all require polarized beam and/or target. The nucleon excitations are of fundamental interest and have been fairly well investigated (at least for the proton) in unpolarized measurements. However, of the 18 response functions upon which the cross section depends, only 4 can be measured in unpolarized experiments. With polarized beam and target one can, in principle, gain access to all 18 response functions (which are combinations of 11 independent helicity amplitudes) and study the spin structure of the nucleon resonances.

The large acceptance of the CLAS detector enabled us to measure multi-particle final states. We studied the single pion production reactions $\gamma p \rightarrow p \pi^{0}$ and $\gamma p \rightarrow n \pi^{+}$with the $\mathrm{NH}_{3}$ target and $\gamma n \rightarrow p \pi^{-}$with the $\mathrm{ND}_{3}$ target. In each case, the exclusive channel was identified using missing mass techniques. After correcting for dilution factor and beam charge asymmetry, we extract $A_{e t}=\sigma_{e t} / \sigma_{0}$ and divide by the kinematic factor $\sqrt{1-\varepsilon^{2}} \cos \theta_{\gamma}$ to obtain the asymmetry $\left(A_{1}+\eta A_{2}\right) /(1+\varepsilon R)$. Figure 4 shows results from the $\pi^{+}$channel [9] as a function of $Q^{2}$ and the polar angle of the emitted pion for various regions in invariant mass $W$. Predictions of the phenomenological models A0 [7] and MAID2000 [10] are shown as the solid and dashed lines. An AO calculation of the asymmetry due to resonances only is shown as the dotted line. Non-resonant contributions are clearly important at low $W$. As an example of the sensitivity of this 

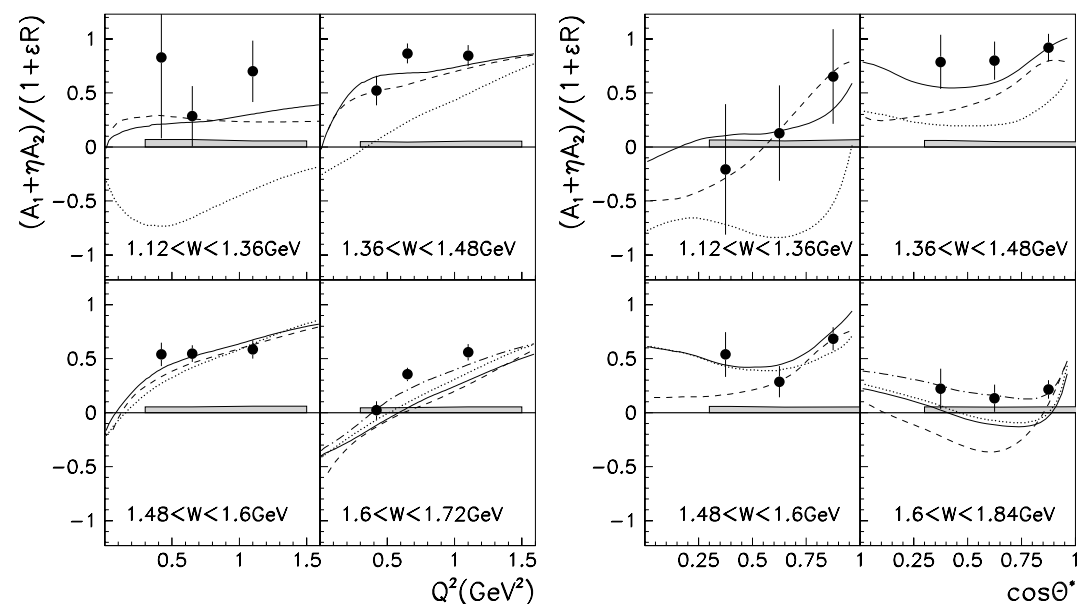

FIGURE 4. The asymmetry $\left(A_{1}+\eta A_{2}\right) /(1+\varepsilon R)$ as a function of $Q^{2}$ (left) and $\cos \theta_{\pi}$ (right) for four different bins in $W$ [9]. See the text for an explanation of the curves.

asymmetry to the details of individual resonance contributions, the asymmetry $A_{1}$ for the $\mathrm{F}_{15}$ (1680) resonance was increased by 0.4 using $\mathrm{AO}$ and plotted as the dot dashed curve in the fourth $W$ panel, resulting in improved agreement with the data. AO also includes a new parametrization for the $\mathrm{S}_{11}(1535)$ in which $A_{1 / 2}$ has been increased, which may explain its better agreement with the data at forward angles. With the vastly improved statistics of the full EG1 data set, we plan to study the resonance region with other exclusive channels $(e . g ., \rho, \omega)$ in addition to single pion production.

\section{ACKNOWLEDGMENTS}

This research is supported by the US Department of Energy under grant DE-FG0296ER40960.

\section{REFERENCES}

1. J.D. Bjorken et al., Phys. Rev. 148, 1467 (1966).

2. S. Gerasimov, Sov. J. Nucl. Phys. 2, 430 (1966); S.D. Drell and A.C. Hearn, Phys. Rev. Lett. 16, 908 (1966).

3. V.D. Burkert and B.L. Ioffe, Phys. Lett. B 296, 223 (1992).

4. J. Soffer and O.V. Teryaev, Phys. Rev. D 51, 25 (1995).

5. K. Abe et al., Phys. Rev. D 58, 112003 (1998).

6. X. Ji and J. Osborne, J. Phys. G 27, 127 (2001).

7. V. Burkert and Z. Li, Phys. Rev. D 47, 46 (1993).

8. K.V. Dharmawardane, these proceedings.

9. R. De Vita et al., Phys. Rev. Lett. 88, 082001 (2002).

10. D. Drechsel et al., Nucl. Phys. A645, 145 (1999). 